Homology, Homotopy and Applications, vol.18(1), 2016, pp.373-380

\title{
THE MULTIPLICATIVE STRUCTURE ON THE GRADED SLICES OF HERMITIAN $K$-THEORY AND WITT-THEORY
}

\author{
OLIVER RÖNDIGS AND PAUL ARNE ØSTVÆR
}

(communicated by Charles A. Weibel)

\begin{abstract}
We give concise formulas in terms of generators and relations for the multiplicative structures on the graded slices of hermitian $K$-theory and Witt-theory.
\end{abstract}

\section{Introduction}

Fix a field $F$ of characteristic $\operatorname{char}(F) \neq 2$ and let $X \in \operatorname{Sm}_{F}$ be a smooth scheme of finite type over $F$. The Grothendieck-Witt group classifying symmetric bilinear forms on $X$ is represented by the hermitian $K$-theory spectrum $\mathbf{K Q}$ in the stable motivic homotopy category $\mathbf{S H}$ of $F[\mathbf{3}]$. Inverting the first Hopf map $\eta$ on $\mathbf{K Q}$ yields the Witt-theory spectrum KT representing the Witt group of $X$. In [9] we computed the slices of KQ and KT in terms of motivic cohomology spectra with integral and mod-2 coefficients, namely

$$
\mathbf{s}_{q}(\mathbf{K Q}) \cong \begin{cases}\Sigma^{2 q, q} \mathbf{M Z} \vee \bigvee_{i<\frac{q}{2}} \Sigma^{2 i+q, q} \mathbf{M Z} / 2 & q \text { even }, \\ \bigvee_{i<\frac{q+1}{2}} \Sigma^{2 i+q, q} \mathbf{M Z} / 2 & q \text { odd }\end{cases}
$$

and

$$
\mathrm{s}_{q}(\mathbf{K T}) \cong \bigvee_{i \in \mathbb{Z}} \Sigma^{2 i+q, q} \mathbf{M Z} / 2
$$

Explicit computations of hermitian $K$-groups in terms of motivic cohomology and a solution of the Milnor conjecture on quadratic forms follow from (1) and (2) via the slice spectral sequence, cf. [9].

The slice filtration introduced in [14] preserves highly structured objects such as algebras and modules $[\mathbf{2}, \mathbf{7}]$. In this paper we set out to refine the computations (1) and (2) by identifying the multiplicative structures of the graded slices $\mathbf{S}_{*}(\mathbf{K Q})$ and $\mathrm{s}_{*}(\mathbf{K T})$. It turns out these can be described by remarkably concise formulas using generators and relations.

As a case in point we consider the multiplication map on the zero slices for Witttheory

The authors acknowledge support from the RCN projects Special Geometries, no. 239015 and Topology, no. 213458.

Received October 26, 2015, revised November 23, 2015; published on May 25, 2016.

2010 Mathematics Subject Classification: 14F42, 19 G99.

Key words and phrases: motivic cohomology and homotopy theory, multiplicative structure on slices of hermitian $K$-theory and Witt-theory spectra.

Article available at http://dx.doi.org/10.4310/HHA.2016.v18.n1.a20

Copyright (C) 2016, International Press. Permission to copy for private use granted. 


$$
\mathrm{s}_{0}(\mathbf{K T}) \wedge \mathrm{s}_{0}(\mathbf{K T}) \longrightarrow \mathrm{s}_{0}(\mathbf{K T}) .
$$

Via the slice computation (2) the map in (3) takes the form

$$
\bigvee_{i, j \in \mathbb{Z}} \Sigma^{2(i+j), 0}(\mathbf{M Z} / 2 \wedge \mathbf{M Z} / 2) \longrightarrow \bigvee_{k \in \mathbb{Z}} \Sigma^{2 k, 0} \mathbf{M Z} / 2
$$

By [4] and [16] the smash product $\mathbf{M Z} / 2 \wedge \mathbf{M Z} / 2$ in (4) identifies with the infinite direct sum

$$
\mathbf{M Z} / 2 \vee \Sigma^{1,0} \mathbf{M Z} / 2 \vee \underset{(m, n) \in I}{\bigvee} \Sigma^{m, n} \mathbf{M Z} / 2
$$

We refer to (10) in $\S 3$ for the precise definition of the index set $I$. The multiplication map in (3) has now been recast as a map between MZ-modules, i.e., as a map between motives [10]. When $k=i+j$, the summand $\mathbf{M Z} / 2$ of (5) maps by the identity to the corresponding summand of $\mathrm{s}_{0}(\mathbf{K T})$ in (3). When $k=i+j+1$, we note that the summand $\Sigma^{1,0} \mathbf{M Z} / 2$ of (5) maps nontrivially under the multiplication map (3); in fact, it maps via the first motivic Steenrod operation $\mathrm{Sq}^{1}$, a.k.a. the Bockstein operator. All the other summands of (5) map trivially under the multiplication map (3) because all the suspensions are of positive weight. This analysis can be bootstrapped to give a description of the multiplicative structure of the graded slices $\mathbf{s}_{*}(\mathbf{K T})$ of Witt-theory in terms of generators and relations involving "twisting" with $\mathrm{Sq}^{1}$ as noted in the above.

In Theorem 3.3 we show the graded slices of hermitian $K$-theory are given by

$$
\mathbf{s}_{*}(\mathbf{K Q}) \cong \mathbf{M Z}\left[\eta, \sqrt{\alpha}^{ \pm 1}\right] /\left(2 \eta=0, \eta^{2} \stackrel{\delta}{\rightarrow} \sqrt{\alpha}\right) .
$$

Here $\eta$ has bidegree $(1,1)$ and $\sqrt{\alpha}$ has bidegree $(4,2)$. As shown in the proof, $\delta$ is the natural connecting map from $\Sigma^{1,0} \mathbf{M Z} / 2$ to $\Sigma^{2,0} \mathbf{M Z}$. Notably, the relation $2 \eta=0$ in (6) is also valid in the coefficients $\mathbf{K O}_{*}$ of the complex points $\mathbf{K O}$ of $\mathbf{K Q}$, i.e., the orthogonal $K$-theory spectrum. However, the relation involving the connecting map $\delta$ cannot be detected on the level of coefficients.

In Theorem 3.6 we show the graded slices of Witt-theory are given by

$$
\mathbf{s}_{*}(\mathbf{K T}) \cong \mathbf{M Z}\left[\eta^{ \pm 1}, \sqrt{\alpha}^{ \pm 1}\right] /\left(2 \eta=2 \sqrt{\alpha}=0, \eta^{2} \stackrel{\mathrm{Sq}^{1}}{\longrightarrow} \sqrt{\alpha}\right) .
$$

All the slices of $\mathbf{K T}$ are $\mathbf{M Z} / 2$-modules. However, the multiplicative relation for $\mathrm{Sq}^{1}$ in (7) shows that neither $\mathbf{s}_{*}(\mathbf{K T})$ nor $\mathbf{s}_{0}(\mathbf{K T})$ are $\mathbf{M Z} / 2$-algebras.

A byproduct of computational interest is a convenient description of the first order differentials along with Leibniz rules in the corresponding slice spectral sequences. We refer to $\S 4$ for precise formulations.

Our arguments take place in the stable motivic homotopy category of $F$, e.g., a ring spectrum refers to a monoid in $\mathbf{S H}$. Throughout the paper we employ the following notation:

\begin{tabular}{l|l}
$F$ & field of characteristic char $(F) \neq 2$ \\
$\operatorname{Sm}_{F}$ & smooth schemes of finite type over $F$ \\
$S^{m, n}, \Omega^{m, n}, \Sigma^{m, n}$ & $(m, n)$-sphere, $(m, n)$-loop space, $(m, n)$-suspension \\
$\mathbf{S H}, \mathbf{S H}^{\text {eff }}$ & motivic and effective motivic stable homotopy categories of $F$ \\
$\mathbf{E}, \mathbf{1}=S^{0,0}$ & generic motivic spectrum, the motivic sphere spectrum \\
$\mathbf{M Z}, \mathbf{M Z} / 2$ & integral and mod-2 motivic cohomology \\
$\mathbf{K G L}, \mathbf{K Q}, \mathbf{K T}$ & algebraic $K$-theory, hermitian $K$-theory, Witt-theory
\end{tabular}




\section{The slice filtration}

Let $i_{q}: \Sigma^{2 q, q} \mathbf{S H}^{\mathrm{eff}} \longrightarrow \mathbf{S H}$ denote the full inclusion of the localizing subcategory

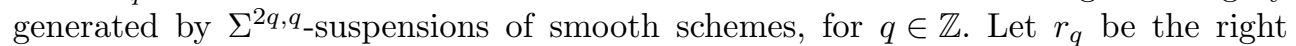
adjoint of $i_{q}$ and set $\mathrm{f}_{q}=i_{q} \circ r_{q}$ as in $[\mathbf{1 4}, \S 2]$. Voevodsky defined the exhaustive slice tower

$$
\cdots \longrightarrow \mathrm{f}_{q+1}(\mathrm{E}) \longrightarrow \mathrm{f}_{q}(\mathrm{E}) \longrightarrow \cdots \longrightarrow \mathrm{f}_{0}(\mathrm{E}) \longrightarrow \mathrm{f}_{-1}(\mathrm{E}) \longrightarrow \cdots \longrightarrow \mathrm{E}
$$

in $\mathbf{S H}$, where $\mathrm{f}_{q}(\mathrm{E}) \rightarrow \mathrm{E}$ is universal for maps $\mathrm{G} \rightarrow \mathrm{E}$ with $\mathrm{G} \in \Sigma^{2 q, q} \mathbf{S H}^{\text {eff }}$. Examples show that the slice tower generalizes the coniveau filtration in algebraic $K$-theory and the filtration by the fundamental ideal in Witt-theory. The $q$ th slice $\mathrm{s}_{q}(\mathrm{E})$ of $\mathrm{E}$ is characterized up to unique isomorphism by the distinguished triangle in $\mathbf{S H}[\mathbf{1 4}$, Theorem 2.2(1)]

$$
\mathrm{f}_{q+1}(\mathrm{E}) \longrightarrow \mathrm{f}_{q}(\mathrm{E}) \longrightarrow \mathrm{s}_{q}(\mathrm{E}) \longrightarrow \Sigma^{1,0} \mathrm{f}_{q+1}(\mathrm{E}) .
$$

It turns out the slice $\mathrm{s}_{q}(\mathrm{E})$ is a module over the motivic ring spectrum $\mathrm{s}_{0}(\mathbf{1})$, cf. $\left[\mathbf{2}, \S 6\right.$ (iv),(v)] and [7, Theorem 3.6.13(6)]. If $F$ is a perfect field, then $\mathrm{s}_{0}(\mathbf{1})$ is the Eilenberg-MacLane spectrum MZ by the works of Levine [5, Theorem 10.5.1] and Voevodsky [17, Theorem 6.6]. By base change this computation extends to all fields; in fact, every field is essentially smooth over a perfect field by [4, Lemma 2.9], and [4, Lemma 2.7(1)] verifies the hypothesis of [8, Theorem 2.12] for an essentially smooth map.

For an axiomatic treatment of the graded slice functor

$$
\mathrm{s}_{*}=\bigoplus \mathrm{s}_{q}: \mathbf{S H} \longrightarrow \mathbf{S H}^{\mathbb{Z}}
$$

we refer to $[\mathbf{2}, \S 2.3]$. Notably, if $E$ is a ring spectrum then $\mathbf{s}_{*}(E)$ is a graded ring spectrum in SH according to [7, Theorem 3.6.13(7)]. In fact, this holds strictly on the level of motivic symmetric spectra by $[\mathbf{2}$, Theorem 5.1], which verifies [5, Conjecture 11.1.1].

Setting $D_{p, q, n}^{1}=\pi_{p, n} \mathrm{f}_{q}(\mathrm{E})$ and $E_{p, q, n}^{1}=\pi_{p, n} \mathrm{~s}_{q}(\mathrm{E})$ gives rise to the tri-graded exact couple:

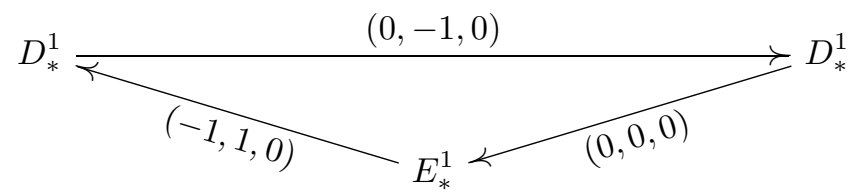

The corresponding slice spectral sequence

$$
E_{p, q, n}^{1} \Longrightarrow \pi_{p, n}(\mathrm{E})
$$

was introduced in $[\mathbf{1 4}, \S 7]$. It is analogous to the Atiyah-Hirzebruch spectral sequence in topology in the sense that its first page is comprised of motivic cohomology groups. Its $d_{1}$-differential

$$
d_{1}^{\mathrm{E}}: \pi_{p, n} \mathrm{~s}_{q}(\mathrm{E}) \longrightarrow \pi_{p-1, n} \mathrm{~s}_{q+1}(\mathrm{E})
$$

is induced by the composite map

$$
\mathrm{d}_{1}^{\mathrm{E}}: \mathrm{s}_{q}(\mathrm{E}) \longrightarrow \Sigma^{1,0} \mathrm{f}_{q+1}(\mathrm{E}) \longrightarrow \Sigma^{1,0} \mathrm{~s}_{q+1}(\mathrm{E}) .
$$

In $\S 4$ we use multiplicative generators for graded slices to give explicit formulas for the $\mathrm{d}_{1}$-differentials in the slice spectral sequences for $\mathbf{K G L}, \mathbf{K Q}$, and $\mathbf{K T}$. 


\section{Multiplicative structure}

The formal difference between the tautological and the trivial line bundle on the projective line $\mathbb{P}^{1}$ defines the motivic Bott map $\beta: S^{2,1} \longrightarrow$ KGL. The composite weak equivalence

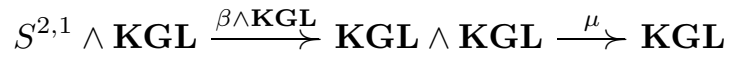

is known as (2,1)-periodicity of KGL [13, Theorem 6.8]. By taking complex points of KGL this yields Bott-periodicity of the unitary $K$-theory spectrum KU. Its coefficients $\mathbf{K U}_{*}$ form a Laurent polynomial algebra over the integers on a generator in degree two. The slices of KGL exhibit a similar property relative to the integral motivic cohomology spectrum. More precisely, Theorem 3.1 follows from the work of Levine $[\mathbf{5}, \S 6.4,11.3]$ and Voevodsky $[\mathbf{1 5}, \mathbf{1 7}]$, augmented by the base change argument to all fields using $[4]$ and $[8]$, cp. $§ 2$.

Theorem 3.1. The graded slices of KGL form a Laurent polynomial ring

$$
\mathrm{s}_{*}(\mathbf{K G L}) \cong \mathbf{M Z}\left[\beta, \beta^{-1}\right] \text {. }
$$

Here $\beta$ is the generator of $\mathbf{s}_{1}(\mathbf{K G L}) \cong \Sigma^{2,1} \mathbf{M Z}$ in bidegree $(2,1)$.

We note the following result relating algebraic and hermitian $K$-theory via the forgetful functor defined in $[\mathbf{9}, \S 3]$. A commutative monoid model for hermitian $K$-theory in $\mathbf{S H}$ was constructed by Panin-Walter in [6], building on work of Schlichting [11].

Theorem 3.2. The hermitian K-theory spectrum KQ is a commutative ring spectrum and the forgetful functor induces a map of motivic ring spectra $\mathbf{K Q} \longrightarrow \mathbf{K G L}$.

The hermitian $K$-theory spectrum is $(8,4)$-periodic by construction $[\mathbf{3}]$ via a map $\alpha: S^{8,4} \longrightarrow \mathrm{KQ}$ inducing a weak equivalence

$$
S^{8,4} \wedge \mathbf{K Q} \stackrel{\alpha \wedge \mathrm{KQ}}{\longrightarrow} \mathrm{KQ} \wedge \mathbf{K Q} \stackrel{\mu}{\longrightarrow} \mathbf{K Q} \text {. }
$$

As in topology, see e.g., $[\mathbf{1}, \S 1.1]$, the composition of the forgetful map $\mathbf{K Q} \longrightarrow \mathbf{K G L}$ with $\alpha$ is $\beta^{4}$. (The referee kindly pointed out that this follows by comparing the projective line bundle formula for $G W_{0}^{[1]}\left(\mathbb{P}^{1}\right)$ with the class $[\mathcal{O}]-[\mathcal{O}(-1)] \in K_{0}\left(\mathbb{P}^{1}\right)$, see $[\mathbf{1 1}, \S 9.4]$.) It follows that the graded slice $s_{*}(\mathbf{K Q})$ contains a Laurent polynomial algebra generated by $\alpha$. In fact, the computation of the slices of $\mathbf{K Q}$ provides a summand $\Sigma^{4,2} \mathbf{M Z}$ of $\mathbf{s}_{2}(\mathbf{K Q})$ which maps by the identity to $\mathrm{s}_{2}(\mathbf{K G L})=\Sigma^{4,2} \mathbf{M Z}$ (see $\left[\mathbf{9}\right.$, Corollary 4.15]). Hence $\mathbf{s}_{*}(\mathbf{K Q})$ contains a Laurent polynomial algebra generated by an element $\sqrt{\alpha}$ of bidegree $(4,2)$ with the property that $\sqrt{\alpha} \cdot \sqrt{\alpha}=\alpha$. The proof of $[\mathbf{9}$, Corollary 4.13] shows the Hopf map $\eta$ generates the top dimensional summand $\Sigma^{1,1} \mathbf{M Z} / 2$ of $\mathrm{s}_{1}(\mathbf{K Q})$. By abuse of notation we shall not distinguish notationally between $\eta$ and the corresponding generator of the slice summand.

We claim there is a relation between $\eta$ and $\sqrt{\alpha}$ : Since the forgetful map $\mathbf{K Q} \longrightarrow$ KGL induces the connecting map $s_{1}(\mathbf{K Q}) \longrightarrow s_{1}(\mathbf{K G L})$ on the top summand, i.e., $\delta: \Sigma^{1,1} \mathbf{M Z} / 2 \longrightarrow \Sigma^{2,1} \mathbf{M Z}$, the multiplication $\mathrm{s}_{1}(\mathbf{K Q}) \wedge \mathrm{s}_{1}(\mathbf{K Q}) \longrightarrow \mathrm{s}_{2}(\mathbf{K Q})$ maps nontrivially to the summand $\Sigma^{4,2} \mathbf{M Z}$. This follows from the commutative diagram: 


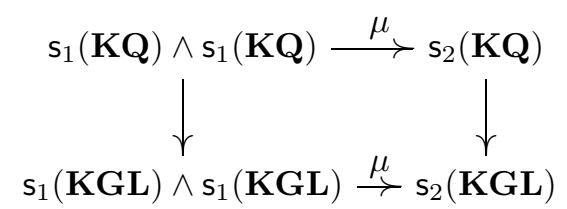

To describe the multiplicative structure, we note that every summand of $\mathbf{s}_{q}(\mathbf{K Q})$ is generated by a product of the form $\sqrt{\alpha}^{m} \eta^{n}$, where $m \in \mathbb{Z}$ and $n \in \mathbb{N}$, with $q=$ $2 m+n$. Such a product generates a copy of $\mathbf{M Z}$ precisely if $n=0$, and a copy of $\mathbf{M Z} / 2$ precisely if $n>0$. It remains to describe the multiplicative structure on the smash powers

$$
\sqrt{\alpha}^{m} \eta^{n} \wedge \sqrt{\alpha}^{p} \eta^{q} .
$$

Under the multiplication on slices it maps to the smash power

$$
\sqrt{\alpha}^{m+p} \eta^{n+q}
$$

and if $n, q$ are positive it also maps via the unique nontrivial map to the summand in degree $4 m+4 p+n+q+2$ generated by

$$
\sqrt{\alpha}^{m+p+1} \eta^{n+q-2} \text {. }
$$

This map can be described explicitly using the smash product decomposition of right MZ/2-modules

$$
\mathbf{M Z} / 2 \wedge \mathrm{MZ} / 2 \stackrel{\sim}{\longrightarrow} \mathrm{MZ} / 2 \vee \Sigma^{1,0} \mathbf{M Z} / 2 \vee \bigvee_{(i, j) \in I} \Sigma^{i, j} \mathbf{M Z} / 2
$$

from $[\mathbf{4}$, Theorem 1.1(3)], [16], where $I \subset \mathbb{N} \times \mathbb{N}$ consists of pairs $(i, j)$ of integers with $i \geqslant 2 j>0$. In terms of the weak equivalence (10) the unit and multiplication maps are given by:

$$
\begin{aligned}
(\mathrm{id}, 0, \ldots): \mathbf{M Z} / 2 & \cong \mathbf{M Z} / 2 \wedge \mathbf{1} \rightarrow \mathbf{M Z} / 2 \wedge \mathbf{M Z} / 2, \\
\left(\mathrm{id}, \mathrm{Sq}^{1}, 0, \ldots\right): \mathbf{M Z} / 2 & \cong \mathbf{1} \wedge \mathbf{M Z} / 2 \rightarrow \mathbf{M Z} / 2 \wedge \mathbf{M Z} / 2, \\
(\mathrm{id}, 0, \ldots): \mathbf{M Z} / 2 & \wedge \mathbf{M Z} / 2 \rightarrow \mathbf{M Z} / 2 .
\end{aligned}
$$

For bidegree reasons, a map from $\mathbf{M Z} / 2 \wedge \mathbf{M Z} / 2$ to either $\Sigma^{2,0} \mathbf{M Z}$ or $\Sigma^{2,0} \mathbf{M Z} / 2$ can be nontrivial only on the summand $\Sigma^{1,0} \mathbf{M Z} / 2$ in (10), and the nontrivial one is the connecting map $\delta$ or $\mathrm{Sq}^{1}=\operatorname{pr} \circ \delta$, respectively. By (9) the slice multiplication map $\mu$ induces the nontrivial map on the summand $\Sigma^{3,2} \mathbf{M Z}$ of $s_{1}(\mathbf{K Q}) \wedge s_{1}(\mathbf{K Q})$ to the summand $\Sigma^{4,2} \mathbf{M Z}$ of $s_{2}(\mathbf{K Q})$. We write $\delta: \eta^{2} \rightarrow \sqrt{\alpha}$ for this relation.

Theorem 3.3. The graded slices of $\mathbf{K Q}$ are given by

$$
\mathbf{s}_{*}(\mathbf{K Q}) \cong \mathbf{M Z}\left[\eta, \sqrt{\alpha}^{ \pm 1}\right] /\left(2 \eta=0, \eta^{2} \stackrel{\delta}{\rightarrow} \sqrt{\alpha}\right) .
$$

Here $\eta$ has bidegree $(1,1)$ and $\sqrt{\alpha}$ has bidegree $(4,2)$.

Recall that $\eta: S^{1,1} \longrightarrow \mathbf{1}$ denotes the Hopf map induced by the canonical map $\mathbb{A}^{2} \backslash\{0\} \longrightarrow \mathbb{P}^{1}$. Every motivic spectrum $\mathrm{E}$ is a module over $\mathbf{1}$, so there is a naturally induced map $\eta: \Sigma^{1,1} \mathrm{E} \longrightarrow \mathrm{E}$. The Witt-theory spectrum $\mathbf{K} \mathbf{T}=\mathbf{K} \mathbf{Q}\left[\eta^{-1}\right]$ is defined by inverting $\eta$ in hermitian $K$-theory. From Theorem 3.2 we can infer the following result.

Corollary 3.4. The Witt-theory spectrum $\mathbf{K T}=\mathbf{K Q}\left[\eta^{-1}\right]$ is a $\mathbf{K Q}$-algebra. 
Let $u: \mathbf{K Q} \longrightarrow \mathbf{K T}$ be the canonical map. We cite from [9, Proposition 4.29] the next result.

Proposition 3.5. Restricting the map $\mathrm{s}_{2 q}(u): \mathrm{s}_{2 q}(\mathbf{K Q}) \longrightarrow \mathrm{s}_{2 q}(\mathbf{K T})$ to the summand $\Sigma^{4 q, 2 q} \mathbf{M Z}$ yields the projection map $\Sigma^{4 q, 2 q} \mathbf{M Z} \longrightarrow \Sigma^{4 q, 2 q} \mathbf{M Z} / 2$ composed with the inclusion into $\mathrm{s}_{2 q}(\mathbf{K T})$. Restricting the same map to a suspension of $\mathbf{M Z} / 2$ yields the inclusion into $\mathbf{s}_{2 q}(\mathbf{K T})$. On slices of odd degree, the map $\mathbf{s}_{2 q+1}(u): \mathbf{s}_{2 q+1}(\mathbf{K Q}) \longrightarrow$ $\mathrm{s}_{2 q+1}(\mathbf{K T})$ is the inclusion.

We note there is an induced $(1,1)$-periodicity isomorphism

$$
\eta: \Sigma^{1,1} \mathbf{K T} \stackrel{\cong}{\cong} \mathbf{T} .
$$

It follows that $\mathbf{s}_{q}(\mathbf{K T}) \cong \Sigma^{q, q} \mathbf{s}_{0}(\mathbf{K T})$, cf. [9, Example 2.3]. The multiplicative structure on the graded slices of Witt-theory follows immediately.

Theorem 3.6. The graded slices of $\mathbf{K T}$ are given by

$$
\mathbf{s}_{*}(\mathbf{K T}) \cong \mathbf{M Z}\left[\eta^{ \pm 1}, \sqrt{\alpha}^{ \pm 1}\right] /\left(2 \eta=2 \sqrt{\alpha}=0, \eta^{2} \stackrel{\mathrm{Sq}^{1}}{\longrightarrow} \sqrt{\alpha}\right) .
$$

Here $\eta$ has bidegree $(1,1)$ and $\sqrt{\alpha}$ has bidegree $(4,2)$.

Remark 3.7. The slices of $\mathbf{K T}$ are $\mathbf{M Z} / 2$-modules. However, the multiplicative relation involving the Steenrod square $\mathrm{Sq}^{1}$ shows that neither $\mathrm{s}_{*}(\mathbf{K T})$ nor $\mathrm{s}_{0}(\mathbf{K T})$ are $\mathrm{MZ} / 2$-algebras.

The multiplicative structure on $\mathrm{s}_{*}(\mathbf{K T})$ in Theorem 3.6 shows that for $X \in \mathrm{Sm}_{F}$, the maps $\left[X_{+}, s_{*}(\mathbf{K T})\right]$ form a graded ring with respect to the multiplication $\star$ given by

$$
\left(a_{m, r}, b_{n, s}\right) \longmapsto(a \cdot b)_{m+n, r+s}+\left(\mathrm{Sq}^{1} a \cdot \mathrm{Sq}^{1} b\right)_{m+n+1, r+s-2} .
$$

Here we write $a \cdot b$ for multiplication of motivic cohomology classes, and $c_{l, t}$ for a generic element in the image of the map

$$
\left[X_{+}, \Sigma^{4 l+t, 2 l+t} \mathbf{M Z} / 2\right] \longrightarrow\left[X_{+}, \mathrm{s}_{2 l+t}(\mathbf{K T})\right]
$$

induced by the inclusion of the summand generated by $\sqrt{\alpha}^{l} \eta^{t}$.

\section{Slice differentials}

The multiplicative structure on the slices of $\mathbf{K Q}$ and $\mathbf{K T}$ allows for very concise and convenient descriptions of the first differentials (8) in the corresponding slice spectral sequences. This follows from and improves on our work in $[\mathbf{9}, \S 5]$. To begin, recall from [9, Lemma 5.1] that the first differential for $\mathbf{K G L} / 2$ is given by

$$
\mathrm{d}_{1}^{\mathrm{KGL} / 2}\left(\beta^{m}\right)=\mathrm{Q}_{1} \beta^{m-1} .
$$

Here $\mathrm{Q}_{1}=\mathrm{Sq}^{2} \mathrm{Sq}^{1}+\mathrm{Sq}^{1} \mathrm{Sq}^{2}$ is the first Milnor operation.

A formula for the first differential of $\mathbf{K Q}$ follows from (11) as in [9, Theorem 5.5]. In terms of generators for the slices we have: 
MULTIPLICATIVE STRUCTURE ON HERMITIAN $K$-THEORY AND WITT-THEORY 379

$$
\begin{aligned}
& \mathrm{d}_{1}^{\mathbf{K Q}}\left(\sqrt{\alpha}^{m} \eta^{n}\right)= \\
& \qquad \begin{array}{ll}
\tau \sqrt{\alpha}^{m-1} \eta^{n+3}+\left(\mathrm{Sq}^{2}+\rho \mathrm{Sq}^{1}\right) \sqrt{\alpha}^{m} \eta^{n+1}+\mathrm{Sq}^{3} \mathrm{Sq}^{1} \sqrt{\alpha}^{m+1} \eta^{n-1} & m \equiv 1(2), n>1, \\
\mathrm{Sq}^{2} \sqrt{\alpha}^{m} \eta^{n+1}+\mathrm{Sq}^{3} \mathrm{Sq}^{1} \sqrt{\alpha}^{m+1} \eta^{n-1} & m \equiv 0(2), n>1, \\
\tau \sqrt{\alpha}^{m-1} \eta^{4}+\left(\mathrm{Sq}^{2}+\rho \mathrm{Sq}^{1}\right) \sqrt{\alpha}^{m} \eta^{2}+\delta \mathrm{Sq}^{2} \mathrm{Sq}^{1} \sqrt{\alpha}^{m+1} & m \equiv 1(2), n=1, \\
\mathrm{Sq}^{2} \sqrt{\alpha}^{m} \eta^{2}+\delta \mathrm{Sq}^{2} \mathrm{Sq}^{1} \sqrt{\alpha}{ }^{m+1} & m \equiv 0(2), n=1, \\
\tau \mathrm{pr} \sqrt{\alpha}^{m-1} \eta^{2}+\mathrm{Sq}^{2} \mathrm{pr} \sqrt{\alpha} m & m \equiv 1(2), n=0, \\
\mathrm{Sq}^{2} \sqrt{\alpha}^{m} \eta^{n+1} & m \equiv 0(2), n=0 .
\end{array}
\end{aligned}
$$

The classes $\tau \in h^{0,1} \cong \mu_{2}(F)$ and $\rho \in h^{1,1} \cong F^{\times} / 2$ are represented by $-1 \in F$; here $h^{p, q}$ is shorthand for the mod-2 motivic cohomology group of $F$ in degree $p$ and weight $q[\mathbf{1 2}, \S 3]$.

In the case of $\mathbf{K T}$, (12) implies along the lines of [9, Theorem 5.3] the formula:

$$
\begin{aligned}
& \mathrm{d}_{1}^{\mathbf{K T}}\left(\sqrt{\alpha}^{m} \eta^{n}\right)= \\
& \begin{cases}\tau \sqrt{\alpha}^{m-1} \eta^{n+3}+\left(\mathrm{Sq}^{2}+\rho \mathrm{Sq}^{1}\right) \sqrt{\alpha}^{m} \eta^{n+1}+\mathrm{Sq}^{3} \mathrm{Sq}^{1} \sqrt{\alpha}^{m+1} \eta^{n-1} & m \equiv 1(2), \\
\mathrm{Sq}^{2} \sqrt{\alpha}^{m} \eta^{n+1}+\mathrm{Sq}^{3} \mathrm{Sq}^{1} \sqrt{\alpha}^{m+1} \eta^{n-1} & m \equiv 0(2) .\end{cases}
\end{aligned}
$$

For $X \in \mathrm{Sm}_{F}$ a straightforward calculation using the Cartan formulas in [4, Theorem 5.1(2)], [16, Theorem 10.2] for $\mathrm{Sq}^{1}$ and $\mathrm{Sq}^{2}$ shows the first differential satisfies the Leibniz rule

$$
d_{1}^{\mathbf{K T}}\left(a_{m, r} \star b_{n, s}\right)=d_{1}^{\mathbf{K T}}\left(a_{m, r}\right) \star b_{n, s}+a_{m, r} \star d_{1}^{\mathbf{K T}}\left(b_{n, s}\right) .
$$

Here the multiplication $\star$ on $\left[X_{+}, s_{*}(\mathbf{K T})\right]$ is defined in $\S 3$.

However, the first differential for $\mathbf{K T}$ does not satisfy the Leibniz rule with respect to the Laurent polynomial ring multiplication

$$
\left(a_{m, r}, b_{n, s}\right) \longmapsto(a \cdot b)_{m+n, r+s} .
$$

An easy counterexample is given by $X=\operatorname{Spec}(F)$ and $a=b=\tau_{0,1}$. The motivic cohomology class

$$
d_{1}^{\mathbf{K T}}\left(\tau_{0,1} \cdot \tau_{0,1}\right)=\mathbf{S q}^{2}\left(\tau_{0,2}^{2}\right)=\left(\tau \rho^{2}\right)_{0,3}
$$

is nonzero if $F$ has a real embedding, while $d_{1}^{\mathbf{K T}}\left(\tau_{0,1}\right)=0$. On the other hand, we have

$$
d_{1}^{\mathbf{K T}}\left(\tau_{0,1} \star \tau_{0,1}\right)=d_{1}^{\mathbf{K T}}\left(\tau_{0,2}^{2}+\rho_{1,0}^{2}\right)=\mathrm{Sq}^{2}\left(\tau_{0,2}^{2}\right)+\left(\tau \rho^{2}\right)_{0,3}=0_{0,3}
$$

\section{References}

[1] A.K. Bousfield. A classification of K-local spectra. J. Pure Appl. Algebra, 66(2):121-163, 1990.

[2] J.J. Gutiérrez, O. Röndigs, M. Spitzweck, and P.A. Østvær. Motivic slices and coloured operads. J. Topol., 5(3):727-755, 2012.

[3] J. Hornbostel. $\mathbb{A}^{1}$-representability of hermitian $K$-theory and Witt groups. Topology, 44(3):661-687, 2005.

[4] M. Hoyois, S. Kelly, and P.A. Østvær. The motivic Steenrod algebra in positive characteristic. arXiv:1305.5690, to appear in: J. Eur. Math. Soc. (JEMS).

[5] M. Levine. The homotopy coniveau tower. J. Topol., 1(1):217-267, 2008. 
[6] I. Panin and C. Walter. On the motivic commutative ring spectrum BO. arXiv: 1011.0650.

[7] P. Pelaez. Multiplicative properties of the slice filtration. Astérisque, 335:xvi+289, 2011.

[8] P. Pelaez. On the functoriality of the slice filtration. J. K-Theory, 11(1):55-71, 2013.

[9] O. Röndigs and P.A. Østvær. Slices of hermitian $K$-theory and Milnor's conjecture on quadratic forms. Geom. Topol., 20(2):1157-1212, 2016.

[10] O. Röndigs and P.A. Østvær. Modules over motivic cohomology. Adv. Math., 219(2):689-727, 2008.

[11] M. Schlichting. Hermitian K-theory, derived equivalences and Karoubi's fundamental theorem. arXiv:1209.0848.

[12] A. Suslin and V. Voevodsky. Bloch-Kato conjecture and motivic cohomology with finite coefficients. In The Arithmetic and Geometry of Algebraic Cycles (Banff, AB, 1998), volume 548 of NATO Sci. Ser. C, Math. Phys. Sci., pages 117-189. Kluwer Acad. Publ., Dordrecht, 2000.

[13] V. Voevodsky. $\mathbb{A}^{1}$-homotopy theory. In Proceedings of the International Congress of Mathematicians, Extra Vol. I (Berlin, 1998), pages 579-604 (electronic), 1998.

[14] V. Voevodsky. Open problems in the motivic stable homotopy theory. I. In Motives, Polylogarithms and Hodge Theory, Part I (Irvine, CA, 1998), volume 3 of Int. Press Lect. Ser., pages 3-34. Int. Press, Somerville, MA, 2002.

[15] V. Voevodsky. A possible new approach to the motivic spectral sequence for algebraic $K$-theory. In Recent Progress in Homotopy Theory (Baltimore, MD, 2000), volume 293 of Contemp. Math., pages 371-379. Am. Math. Soc., Providence, RI, 2002.

[16] V. Voevodsky. Reduced power operations in motivic cohomology. Publ. Math. Inst. Hautes Étud. Sci., 98:1-57, 2003.

[17] V. Voevodsky. On the zero slice of the sphere spectrum. Tr. Mat. Inst. Steklova, 246(Algebr. Geom. Metody, Svyazi i Prilozh.):106-115, 2004.

Oliver Röndigs oroendig@uni-osnabrueck.de

Institut für Mathematik, Universität Osnabrück, Albrechtstr. 28a, 49076 Osnabrück, Germany

Paul Arne Østvær paularne@math.uio.no

Department of Mathematics, University of Oslo, P.O. Box 1053 Blindern, 0316 Oslo, Norway 